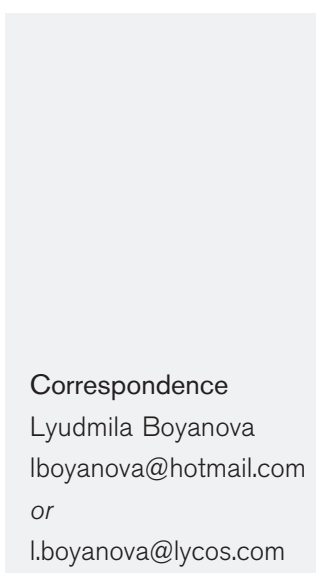

Received 21 January 2007

Accepted 4 April 2007

\section{Helicobacter pylori and Helicobacter heilmannii in untreated Bulgarian children over a period of 10 years}

\author{
Lyudmila Boyanova, ${ }^{1}$ Elena Lazarova, ${ }^{2}$ Christo Jelev, ${ }^{2}$ Galina Gergova ${ }^{1}$ \\ and Ivan Mitov ${ }^{1}$ \\ ${ }^{1}$ Department of Microbiology, Medical University of Sofia, Zdrave Street 2, 1431 Sofia, Bulgaria \\ ${ }^{2}$ Department of Gastroenterology, University Paediatric Hospital, Sofia, Bulgaria
}

\begin{abstract}
The aims of the study were to evaluate the incidence of Helicobacter pylori and Helicobacter heilmannii in untreated Bulgarian children from 1996 to 2006, to analyse the performance of diagnostic tests, and to look at $H$. pylori density in specimens by culture. Antral specimens from children with chronic gastritis $(n=513)$, peptic ulcers $(n=54)$ and other diseases $(n=91)$ were evaluated by direct Gram staining (DGS), in-house rapid urease test (RUT) and culture. The living environment and semi-quantitative $H$. pylori density were assessed in 188 and 328 children, respectively. H. pylori infection was found in children with ulcers (77.8\%), chronic gastritis (64.5 $\%$ ) and other diseases (36.3\%). Half (51.4\%) of patients aged 1-5 years and $77.4 \%$ of those aged 16-17 years were $H$. pylori-positive. Of all children, 328 (49.8\%) showed positive DGS, $184(28 \%)$ had a positive RUT, and 386 (58.7\%) were culture-positive. Unlike gastric mucus specimens, frozen biopsy specimens provided reliable diagnosis. H. heilmannii was observed in two $(0.3 \%)$ children. High H. pylori density (growth into all quadrants of plates) was found in $18 \%$ of 328 children evaluated, involving $31 \%$ of ulcer and $16.7 \%$ of non-ulcer patients. H. pylori infection was more common in rural children with chronic gastritis $(91.3 \%)$ than in the remainder $(66.7 \%)$. In conclusion, H. pylori infection was common in symptomatic Bulgarian children. The infection prevalence was $>77 \%$ in patients aged 16-17 years, in children with a duodenal ulcer, and in rural patients. H. heilmannii infection was uncommon. The performance of the bacterial culture was good. The impact of $H$. pylori density on the clinical expression and eradication of the infection requires further evaluation. The results highlight the need for routine $H$. pylori diagnosis in rural children with chronic gastritis.
\end{abstract}

\section{INTRODUCTION}

Helicobacter pylori infection is associated with gastroduodenal diseases in humans (Matysiak-Budnik et al., 2006). The incidence of the infection in children ranges from 10 to $80 \%$, and is high in developing and some other countries (Torres, 2000; Janulaityte-Gunther et al., 2005; Kato et al., 2004). H. pylori infection is most often intrafamilial and spreads by oral-oral or faecal-oral transmission (Megraud, 2003; Mladenova et al., 2006). Helicobacter heilmannii can also infect the human stomach. The incidence (usually $<1 \%$ ) of this zoonotic infection in dyspeptic patients is much lower than that caused by $H$. pylori (Ierardi et al., 2001; Okiyama et al., 2005). There are, however, only a few reports concerning $H$. heilmannii incidence in children (Coman et al., 1996; Mention et al., 1999; Sykora et al., 2004).

Abbreviations: DGS, direct Gram staining; RUT, rapid urease test.
The aims of the present study were to assess the incidence and characteristics of $H$. pylori and $H$. heilmannii infections in untreated Bulgarian children over a period of 10 years, according to age, sex, disease and living environment; to analyse the performance of diagnostic tests according to the type of antral specimen; and to evaluate $H$. pylori density in the biopsy specimens by culture.

\section{METHODS}

Patients and specimens. In total, 658 consecutive, untreated children with upper gastrointestinal complaints were evaluated from January 1996 to November 2006. Informed written consent was obtained from the parents of all the children. The patients were aged $1-5$ years $(n=37), 6-10$ years $(n=216), 11-15$ years $(n=272)$ and $16-17$ years $(n=133)$. The classification of patients' diseases was based on endoscopic findings and clinical signs (Kalach et al., 2005). The children suffered from chronic gastritis $(n=513)$, duodenal ulcers $(n=50)$, gastric ulcers $(n=4)$, other gastroduodenal diseases $(n=36)$ and non-ulcer dyspepsia $(n=55)$. 
One antral biopsy specimen per child was taken from 585 children and gastric mucus specimens were taken from 73 children. Both antral biopsy and gastric mucus specimens were taken from five children. The mucus specimens were taken by a brush that was introduced into the stomach through the biopsy channel of the endoscope, and then the antral mucosal surface was stroked with the brush. All the specimens were transported in Stuart transport medium (Becton Dickinson). In total, 622 fresh specimens were transported in Stuart transport medium for $<5 \mathrm{~h}$, and 36 specimens were frozen in Stuart transport medium at $-70{ }^{\circ} \mathrm{C}$ for $1-7$ days (15 specimens) or for $8-16$ days ( 21 specimens).

Microbiology. The specimens were used for direct Gram staining (DGS), in-house rapid urease test (RUT) and culture. Each specimen was divided into three parts. A smear was prepared from one part of the specimen by scraping the biopsy onto a slide. The smear was fixed and used for a modified Gram stain with carbol fuchsin as the counterstain. For performing the RUT, one-third of the biopsy specimen was placed in urea agar medium with $10 \%$ urea. The RUT was prepared using urea agar base (Merck) by adding the filtersterilized urea (Merck) after the agar had been autoclaved and cooled to $48{ }^{\circ} \mathrm{C}$. Clinical strains of Proteus mirabilis and Escherichia coli were used as a positive and negative control, respectively. The RUT was incubated at $35{ }^{\circ} \mathrm{C}$. Observations for colour change were made after $30 \mathrm{~min}$ and $3 \mathrm{~h}$. The remaining part of the specimen was homogenized in $0.1 \mathrm{ml}$ sterile saline using sterile needles, and the homogenate was used for culture.

The culture was performed on Columbia agar (Becton Dickinson) with $10 \%$ sheep blood (non-selective medium) and the same medium with $1 \%$ Isovitalex (Becton Dickinson) and the following agents: $10 \mathrm{mg}$ vancomycin $\mathrm{ml}^{-1}, 5 \mathrm{mg}$ trimethoprim $\mathrm{ml}^{-1}, 5 \mathrm{mg}$ cefsulodin $\mathrm{ml}^{-1}$ and $5 \mathrm{mg}$ amphotericin $\mathrm{B} \mathrm{ml}^{-1}$ (selective medium). One selective and one non-selective medium plate were used for the primary culture of specimens. Plates were incubated at $35^{\circ} \mathrm{C}$ for up to 11 days in a microaerophilic atmosphere (Campy Pak; Becton Dickinson). Identification of $H$. pylori was made by Gram staining of the colonies, lack of aerobic growth on blood agar plates, and testing for the presence of urease, oxidase and catalase. The specimens were considered $H$. pylori-positive if the culture was positive, or in case of negative culture, if the two other tests used (DGS and RUT) were both positive. The performance of the tests was evaluated.

H. pylori growth in the antral biopsy specimens of 328 consecutive children was expressed semi-quantitatively (as the density of $H$. pylori) as follows: no growth, score 0 ; sparse density with growth into the first or first and second quadrants of the plate, score 1; moderate density with growth into the third quadrant, score 2 ; and high density with growth into all quadrants of the plate, score 3. Both media were considered for scoring the $H$. pylori density, and in case of different scores, the higher one was taken into account (Lai et al., 2003). The living environment was assessed in 188 children, with 161 children living in towns (including 79 patients from the capital) and 27 children living in villages.

H. heilmannii was recognized by Gram smears as corkscrew-shaped bacteria larger than $H$. pylori. The bacteria were not cultured from the specimens, whereas urease was positive after $24 \mathrm{~h}$ incubation.

Statistical analysis. Differences between groups were evaluated by the chi-square test, with or without Yates' correction.

\section{RESULTS AND DISCUSSION}

H. pylori infection was detected in $406(61.7 \%)$ of the untreated Bulgarian children with gastroduodenal diseases. The infection was common even in the youngest patients, with about half ( $51.4 \%, 19$ of 37$)$ of the children aged $1-5$ years being $H$. pylori-positive.

The overall infection incidence in Bulgarian paediatric patients was higher than that (25.6-30.5\%) reported in some European countries and China (Koletzko et al., 2003; Wong et al., 2005; Sykora et al., 2004), but was lower than that in Lithuanian children (79\%; Janulaityte-Gunther et al., 2005). In the present study, more duodenal ulcer patients $(80 \%)$ were $H$. pylori-positive than were those with chronic gastritis $(64.5 \%, P<0.05)$ and the other patients $(36.8 \%, P<0.001)$ (Table 1$)$. However, H. pylori incidence in children with chronic gastritis was higher than that $(25.6 \%)$ reported in a recent study (Wong et al., 2005). The results of the present study were similar to those of a Japanese study (Kato et al., 2004).

In two $(0.3 \%)$ children, $H$. heilmannii was diagnosed by a Gram-stained smear. The patients were an 11-year-old girl with erosive gastritis and a 13-year-old boy with chronic non-erosive gastritis. Both children were from towns and were $H$. pylori-negative. $H$. heilmannii was seen in the Gram-stained biopsy specimens and differed from $H$. pylori by its four to eight visible spirals and larger size. The urease test was positive after $24 \mathrm{~h}$ incubation. There are still only a few reports on $H$. heilmannii incidence in children. The present study demonstrated a slightly lower incidence of $H$. heilmannii in paediatric patients than that reported by other authors (0.4-1.1\%) (Coman et al., 1996; Mention et al., 1999; Sykora et al., 2004).

Increasing age has been associated significantly with the higher risk of $H$. pylori infection in symptomatic children (Wong et al., 2005). Likewise, in the present study, $H$. pylori infection was less common (51.4 \%, 19 of 37 cases) in children aged 1-5 years than in those aged 16-17 years (77.4\%, 103 of 133; $P<0.01)$. Although a male predominance of $H$. pylori infection has been observed in adults, the role of gender as a risk factor for the infection, especially in children, is still debated (de Martel \& Parsonnet, 2006). In the present work, there was no difference in the infection incidence between boys $(62.9 \%$, 175 of 278 patients) and girls $(60.8 \%, 231$ of $380 ; P$ $>0.20$ ).

In rural patients, the overall incidence of the infection (88.9 \%, 24 of 27 patients) was higher than that (65.8\%, 106 of $161 ; P<0.05)$ in the other children. A similar difference in the infection incidence was observed between rural children with chronic gastritis $(91.3 \%, 21$ of 23 patients) and the rest (66.7\%, 94 of $141 ; P<0.05)$. These results could have been due to several reasons, such as a higher incidence of $H$. pylori infection, or lower antibiotic usage in rural children than in those from towns, as well as differences in health-care-seeking behaviour or referral for endoscopy of the paediatric patients.

Of all children, 328 (49.8\%) had positive DGS, 184 (28\%) had positive RUT, and $386(58.7 \%)$ had positive culture. The specimens from $13(2 \%)$ of the 658 children, nine 
Table 1. Clinical and laboratory findings in 658 untreated children with gastroduodenal diseases

\begin{tabular}{|lrcccc|}
\hline Groups of patients & $\begin{array}{c}\text { No. of } \\
\text { patients }\end{array}$ & $\begin{array}{c}\text { No. of } \boldsymbol{H} \text {. } \\
\text { pylori-positive }\end{array}$ & $\begin{array}{c}\text { Percentage } \\
\text { positive }\end{array}$ & 95\% CI & P value \\
\hline Endoscopy & & & & & \\
$\quad$ Gastric ulcer & 4 & 2 & 50.0 & $0-100.0$ & \\
$\quad$ Duodenal ulcer & 50 & 40 & 80.0 & $68.8-91.2$ & $P<0.05$ \\
Chronic gastritis & 513 & 331 & 64.5 & $60.4-68.6$ & \\
Other diseases* & 36 & 15 & 41.7 & $25.4-58.0$ & \\
$\quad$ Non-ulcer dyspepsia & 55 & 18 & 32.7 & $20.2-45.2$ & \\
Age (years) & & & & & \\
1-5 & 37 & 19 & 51.4 & $35.1-67.7$ & \\
6-10 & 216 & 121 & 56.0 & $49.4-62.6$ & \\
11-15 & 272 & 163 & 59.9 & $54.1-65.7$ & \\
16-17 & 133 & 103 & 77.4 & $70.3-84.5$ & \\
Sex & & & & & \\
Boys & 278 & 175 & 62.9 & $57.2-68.6$ & \\
Girls & 380 & 231 & 60.8 & $55.9-65.7$ & \\
Total & 658 & 406 & 61.7 & $58.0-65.4$ & \\
\hline
\end{tabular}

${ }^{\star}$ Reflux oesophagitis and single cases of hiatal hernia, iron deficiency anaemia and Crohn's disease.

(1.5\%) of 585 antral biopsy specimens and four $(5.5 \%)$ of 73 antral mucus specimens, were positive by RUT and negative by both DGS and culture. In addition, the specimens from $44(6.7 \%)$ of all 658 children, involving $37(6.3 \%)$ of the antral biopsy specimens and seven (9.6 $\%)$ of the antral mucus specimens, were positive by DGS and negative by both RUT and culture. According to diagnostic criteria in the present study, if only the DGS or RUT was positive, the specimen was considered $H$. pylorinegative. Culture exhibited the best performance among the diagnostic methods in the present study. This could have been due to the use of both selective and non-selective media with a long incubation (up to 11 days) of the specimens, or to the use of only one-third of a specimen per child for the RUT. The RUT can give false-negative scores with small numbers of $H$. pylori (De Korwin, 2003). Therefore, we recommend evaluating two gastric biopsy specimens per child, in order to optimize the diagnosis of H. pylori infection. According to Ogata et al. (2001), 5-10 $\%$ of $H$. pylori-infected children and adolescents have been misdiagnosed with a single antral-biopsy-based test.

H. pylori infection was detected in $387(62.2 \%)$ of the 622 fresh antral specimens, compared with 19 (52.8\%) of the 36 frozen specimens $(P>0.20)$. Eight $(53.3 \%)$ of 15 specimens frozen for $1-7$ days and $11(52.4 \%)$ of 21 long-term-frozen specimens were positive for $H$. pylori. However, H. pylori infection was detected in significantly more gastric biopsies (63.9\%, 374 of 585 specimens) than gastric mucus specimens $(43.8 \%, 32$ of 73 specimens; $P$ $<0.001)$. The mucus specimens may have included smaller sample volumes than the biopsy specimens, which led to a lower diagnostic performance. By evaluating only the five children with both gastric biopsy and gastric mucus specimens, H. pylori was cultured from biopsy specimens of three of the children but from the antral mucus specimen of only one child. Therefore, the frozen biopsy specimens provided acceptable $H$. pylori detection, while the gastric mucus specimens were not suitable for $H$. pylori diagnosis in paediatric patients. The number of positive results for DGS, culture and RUT in gastric mucus specimens decreased by $22.2,19.8$ and $9.9 \%$, respectively, compared to those in antral biopsy specimens (Table 2). However, Minami et al. (2006) have reported that combination of the endoscopic brushing technique with the loop-mediated isothermal amplification method is a useful and safe system for identifying $H$. pylori infection.

H. pylori density has been found to correlate with the degree of gastric-mucosal inflammation in children (Elitsur et al., 2002). Different authors have used different methods for scoring $H$. pylori density, including histology, culture, real-time quantitative PCR-based techniques or the urea breath test (Elitsur et al., 2002; Lai et al., 2003; Atherton et al., 1996; Abbas et al., 2001; He et al., 2002). One disadvantage of the invasive tests can be the patchy

Table 2. Comparison of diagnostic tests for $H$. pylori infection in 658 untreated Bulgarian paediatric patients

\begin{tabular}{|lrrr|}
\hline \multirow{2}{*}{ Specimen (no.) } & \multicolumn{3}{c|}{ No. (\%) positive by: } \\
\cline { 2 - 4 } & \multicolumn{1}{c}{ DGS } & \multicolumn{1}{c|}{ RUT } & Culture \\
\hline Antral biopsy (585) & $306(52.3)$ & $170(29.1)$ & $356(60.9)$ \\
Antral mucus (73) & $22(30.1)$ & $14(19.2)$ & $30(41.1)$ \\
Total (658) & $328(49.8)$ & $184(28.0)$ & $386(58.7)$ \\
\hline
\end{tabular}




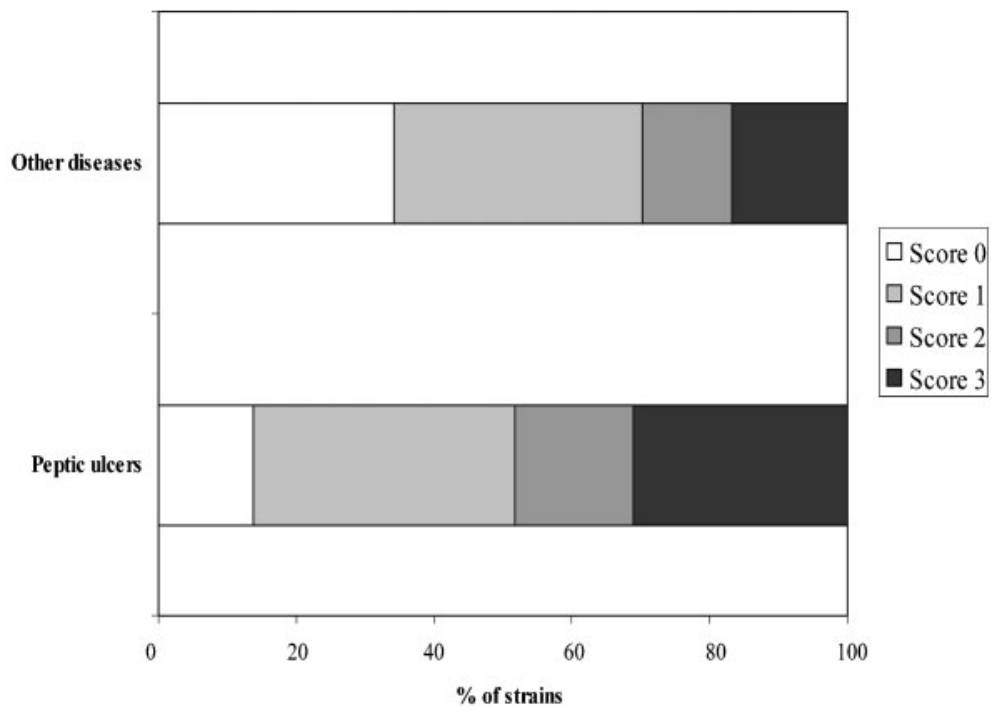

Fig. 1. H. pylori density assessed by semiquantitative culture in 257 untreated paediatric patients.

distribution of $H$. pylori in gastric mucosa (Vinette et al., 2004); however, $H$. pylori density has often been evaluated by invasive tests. In the present study, by semi-quantitative scoring of $H$. pylori growth by culture, the infection in 59 $(18 \%)$ of 328 children evaluated was associated with high H. pylori density (score 3), and that in $103(31.4 \%$ ) of the children was associated with either moderate (score 2 ) or high $H$. pylori density. In addition to cagA and other virulence markers of $H$. pylori strains, bacterial density can also contribute to a more severe infection (Gallo et al., 2003). According to the present results, slightly more ulcer patients ( $31 \%, 9$ of 29 patients) had high $H$. pylori density in the antral biopsy specimens, compared with those with other diseases $(16.7 \%, 50$ of 299 patients; $P>0.05)$ (Fig. 1).

In conclusion, H. pylori infection was common in symptomatic Bulgarian children, and the infection incidence was associated with increasing age, duodenal ulcer and rural living environment, but not with patient gender. In contrast, $H$. heilmannii infection was uncommon. Culture showed a good performance. Frozen biopsy specimens provided reliable $H$. pylori detection, unlike the gastric mucus specimens. Both moderate and high $H$. pylori densities were found in about one-third of the children. The impact of $H$. pylori density, assessed by semiquantitative culture, on the clinical expression of the infection and the success of eradication requires further evaluation. The present results demonstrate the benefit of diagnosing $H$. pylori infection in rural children with chronic gastritis.

\section{REFERENCES}

Abbas, Z., Fareed, R., Baig, M. N., Khan, T. N. \& Shah, M. A. (2001). Prevalence of histological reflux oesophagitis in $H$. pylori positive patients: effect of density of $H$. pylori and activity of inflammation. $J$ Pak Med Assoc 51, 36-41.
Atherton, J. C., Tham, K. T., Peek, R. M., Jr, Cover, T. L. \& Blaser, M. J. (1996). Density of Helicobacter pylori infection in vivo as assessed by quantitative culture and histology. J Infect Dis 174, 552-556.

Coman, G., Burlea, M., Dahorea, C., Mihaila, D. \& Andries, A. (1996). Helicobacter heilmanii gastritis: the bacteriological, endoscopic and histopathological considerations in 18 cases of infection in children. Rev Med Chir Soc Med Nat Iasi 100, 138-142 (in Romanian).

De Korwin, J. D. (2003). Advantages and limitations of diagnostic methods for H. pylori infection. Gastroenterol Clin Biol 27, 380-390 (in French).

de Martel, C. \& Parsonnet, J. (2006). Helicobacter pylori infection and gender: a meta-analysis of population-based prevalence surveys. Dig Dis Sci 51, 2292-2301.

Elitsur, Y., Lawrence, Z. \& Triest, W. E. (2002). Distribution of Helicobacter pylori organisms in the stomachs of children with $H$. pylori infection. Hum Pathol 33, 1133-1135.

Gallo, N., Zambon, C. F., Navaglia, F., Basso, D., Guariso, G., Grazia Piva, M., Greco, E., Mazza, S., Fogar, P. \& other authors (2003). Helicobacter pylori infection in children and adults: a single pathogen but a different pathology. Helicobacter 8, 21-28.

He, Q., Wang, J. P., Osato, M. \& Lachman, L. B. (2002). Real-time quantitative PCR for detection of Helicobacter pylori. J Clin Microbiol 40, 3720-3728.

lerardi, E., Monno, R. A., Gentile, A., Francavilla, R., Burattini, O., Marangi, S., Pollice, L. \& Francavilla, A. (2001). Helicobacter heilmannii gastritis: a histological and immunohistochemical trait. $J$ Clin Pathol 54, 774-777.

Janulaityte-Gunther, D., Kucinskiene, R., Kupcinskas, L., Pavilonis, A., Labanauskas, L., Cizauskas, A., Schmidt, U., Wadstrom, T. \& Andersen, L. P. (2005). The humoral immune response to Helicobacter pylori infection in children with gastrointestinal symptoms. FEMS Immunol Med Microbiol 44, 205-212.

Kalach, N., Mention, K., Guimber, D., Michaud, L., Spyckerelle, C. \& Gottrand, F. (2005). Helicobacter pylori infection is not associated with specific symptoms in nonulcer-dyspeptic children. Pediatrics $115,17-21$.

Kato, S., Nishino, Y., Ozawa, K., Konno, M., Maisawa, S., Toyoda, S., Tajiri, H., Ida, S., Fujisawa, T. \& linuma, K. (2004). The prevalence of Helicobacter pylori in Japanese children with gastritis or peptic ulcer disease. J Gastroenterol 39, 734-738. 
Koletzko, S., Konstantopoulos, N., Bosman, D., Feydt-Schmidt, A., van der Ende, A., Kalach, N., Raymond, J. \& Rüssmann, H. (2003). Evaluation of a novel monoclonal enzyme immunoassay for detection of Helicobacter pylori antigen in stool from children. Gut 52, 804-806.

Lai, Y. C., Wang, T. H., Huang, S. H., Yang, S. S., Wu, C. H., Chen, T. K. \& Lee, C. L. (2003). Density of Helicobacter pylori may affect the efficacy of eradication therapy and ulcer healing in patients with active duodenal ulcers. World J Gastroenterol 9, 1537-1540.

Matysiak-Budnik, T., Laszewicz, W., Lamarque, D. \& Chaussade, S. (2006). Helicobacter pylori and non-malignant diseases. Helicobacter 11 (Suppl. 1), 27-31.

Megraud, F. (2003). When and how does Helicobacter pylori infection occur? Gastroenterol Clin Biol 27, 374-379 (in French).

Mention, K., Michaud, L., Guimber, D., Martin De Lasalle, E., Vincent, P., Turck, D. \& Gottrand, F. (1999). Characteristics and prevalence of Helicobacter heilmannii infection in children undergoing upper gastrointestinal endoscopy. J Pediatr Gastroenterol Nutr 29, 533-539.

Minami, M., Ohta, M., Ohkura, T., Ando, T., Torii, K., Hasegawa, T. \& Goto, H. (2006). Use of a combination of brushing technique and the loop-mediated isothermal amplification method as a novel, rapid, and safe system for detection of Helicobacter pylori. J Clin Microbiol 44, 4032-4037.
Mladenova, I., Durazzo, M. \& Pellicano, R. (2006). Transmission of Helicobacter pylori: are there evidences for a fecal-oral route? Minerva Med 97, 15-18.

Ogata, S. K., Kawakami, E., Patricio, F. R., Pedroso, M. Z. \& Santos, A. M. (2001). Evaluation of invasive and non-invasive methods for the diagnosis of Helicobacter pylori infection in symptomatic children and adolescents. Sao Paulo Med J 119, 67-71.

Okiyama, Y., Matsuzawa, K., Hidaka, E., Sano, K., Akamatsu, T. \& Ota, H. (2005). Helicobacter heilmanniiinfection: clinical, endoscopic and histopathological features in Japanese patients. Pathol Int 55, 398-404.

Sykora, J., Hejda, V., Varvarovska, J., Stozicky, F., Siala, K. \& Schwarz, J. (2004). Helicobacter heilmannii gastroduodenal disease and clinical aspects in children with dyspeptic symptoms. Acta Paediatr 93, 707-709.

Torres, J. (2000). Epidemiologic and clinical aspects of Helicobacter pylori infection in children. Rev Gastroenterol Mex 65 (Suppl. 2), 1319 (in Spanish).

Vinette, K. M., Gibney, K. M., Proujansky, R. \& Fawcett, P. T. (2004). Comparison of PCR and clinical laboratory tests for diagnosing $H$. pylori infection in pediatric patients. BMC Microbiol 4, 5 .

Wong, K. K., Chung, P. H., Lan, L. C., Lin, S. C. \& Tam, P. K. (2005). Trends in the prevalence of Helicobacter pylori in symptomatic children in the era of eradication. J Pediatr Surg 40, 1844-1887. 